\title{
Imaging Ventral Cell Plate Formation in Guard Cells
}

\author{
Xingyun Qi ${ }^{1}$, Keiko U. Torii ${ }^{1,2}$ \\ 1. Department of Biology, University of Washington, Seattle, WA, 98195, USA. \\ ${ }^{2}$ Howard Hughes Medical Institute, University of Washington, Seattle, WA, 98195, USA.
}

Plant cells, unlike animal cells, are encapsulated in rigid cell walls, which provide the mechanical support but also limit the movement of plant cells. Among the few plant cell types that could move, stomatal guard cells are of great importance. The opening and closure of stomatal guard cells control the trade-off between efficient gas exchange for photosynthesis and water loss via transpiration [Figure 1A]. Therefore, an interesting question is to understand on molecular basis the regulation of the ventral cell wall movement of the guard cells.

By analyzing the transcriptome in meristemoid, a stomatal precursor with stem-cell-like properties [Figure 1B-1D], we identified a novel gene with unknown function [1]. The GFP fusion of this gene product driven by its endogenous promoter is specifically expressed in the stomata lineage cells at the late developmental stage from late meristemoids to mature guard cells, with peak expression at the guard mother cells. Interestingly, besides some intracellular highly mobile punctae, the GFP signal is extremely enriched in the new cell plate between two future guard cells. Furthermore, we noted that this protein exhibits a polar distribution at the cell periphery, where the future cell plate will eventually fuse, well in advance of the cytokinesis in guard mother cells. The closest homologue of this novel gene based on DNA alignment is found in the plasmodesmal proteome. Plasmodesmata, the physical channel between two adjacent cells, is formed during cytokinesis [2]. It is known that the plasmodesmata flux could be regulated through dilation/constriction of the neck aperture by the modification of the gatekeeper of plasmadesmata, callose [3,4]. Although continuous plasmodesmata were observed in immature guard cells, no continuous plasmodesmata were found connecting guard cells to sister guard cells or to adjacent epidermal cells [5-7], indicating they were sealed during the maturation of guard cells. We are currently testing our hypothesis that this protein may be involved in ventral cell plate formation as well as plasmodesmata regulation during stomata differentiation.

\section{References:}

[1] LJ Pillitteri et al, Plant Cell. 23(2011), p. 3260

[2] AJ Maule, Curr. Opin. Plant Biol. 11(2008), p. 680

[3] JE Radford, M Vesk and RL Overall, Protoplasma 201(1998), p. 30.

[4] A Levy, D Guenoune-Gelbart and BEpel, Plant Signal. Behav. 2(2007), p. 404.

[5] JE Pallas Jr and HH Mollenhauer, Science 175(1972),p. 1275.

[6] AC Wille andWJ Lucas,Planta160(1984), p, 129.

[7] BA Palevitz andPK Hepler,Planta164(1985), p. 473.

[8] The authors acknowledge funding from a grant from Gordon and Betty Moore Foundation (GBMF3035) to KUT. KUT is an HHMI-GBMF Investigator. 

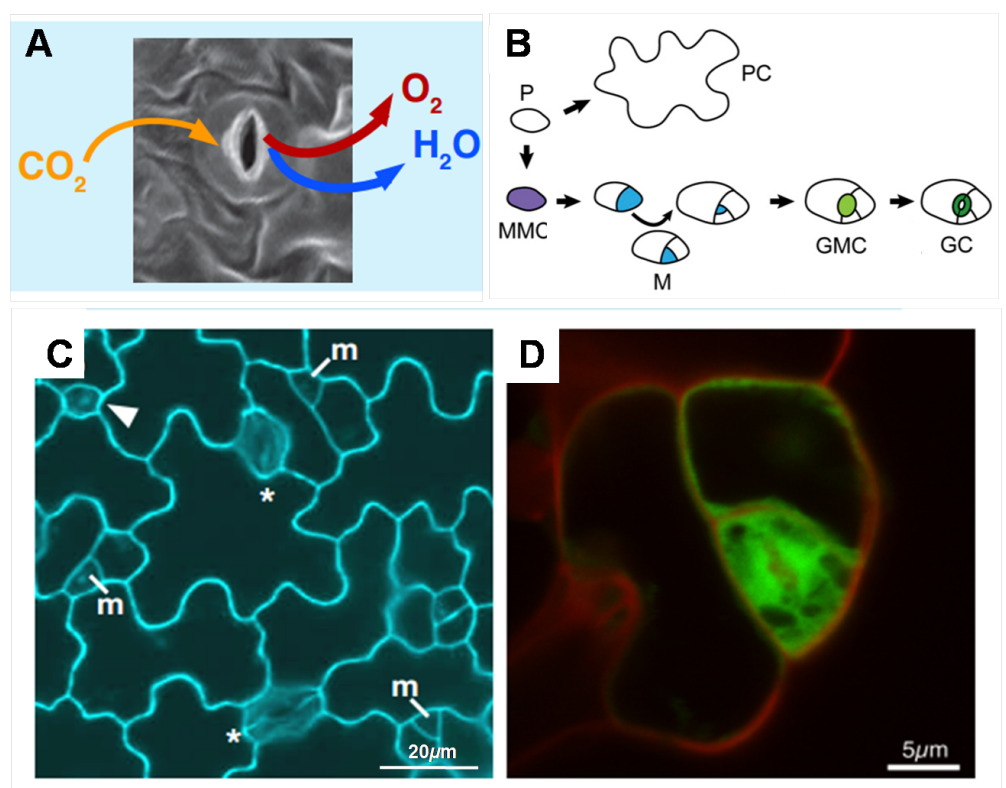

Figure 1. Stomatal development

(A) A stoma is a passage for carbon dioxide, oxygen, and water vapor. Shown is a mature Arabidopsis stoma. (B) A protodermal cell can differentiate into a pavement cell or undergo a transition to be a meristemoid mother cell (MMC). An MMC will create a stomatal precursor stem cell called meristemoid $(\mathrm{M})$ by the initial asymmetric cell division. The $\mathrm{M}$ reiterates one to three rounds of asymmetric amplifying divisions in an inward-spiral manner before differentiating into a guard mother cell (GMC). The GMC devides symmetrically to produce the paired guard cells surrounding a pore, which form a mature stoma. (C) Wild-type leaf epidermis of Arabidopsis. m, meristemoid; arrowhead, guard mother cell; asterisks, mature stoma. (D) A meristemoid (expressing GFP, green) reiterates asymmetric division and renews itself. 